$45 \mid 2010$

Langue française, identité(s) et école(s) : le cas de la minorité catholique au Levant (milieu XIX ${ }^{\mathrm{e}}-\mathrm{XX}^{\mathrm{e}}$

siècles). Représentations identitaires et apprentissage des langues : Europe, bassin méditerranéen ( $\mathrm{XVI}{ }^{\mathrm{e}}-\mathrm{XX}{ }^{\mathrm{e}}$ siècles)

\title{
André Kaspi (sous la direction de). Histoire de l'Alliance israélite universelle de 1860 à nos jours.
}

Paris : Armand Colin, 2010, 575 p. ISBN 978-2-200-35479-4.

Danielle Omer

\section{OpenEdition}

Édition électronique

URL : https://journals.openedition.org/dhfles/2491

DOI : $10.4000 /$ dhfles. 2491

ISSN : 2221-4038

Éditeur

Société Internationale pour l'Histoire du Français Langue Étrangère ou Seconde

Édition imprimée

Date de publication : 1 juin 2010

Pagination : 224-232

ISSN : 0992-7654

Référence électronique

Danielle Omer, «André Kaspi (sous la direction de). Histoire de l'Alliance israélite universelle de 1860 à nos jours. ", Documents pour l'histoire du français langue étrangère ou seconde [En ligne], 45 | 2010, mis en ligne le 31 août 2013, consulté le 27 mai 2021. URL : http://journals.openedition.org/dhfles/2491 DOI : https://doi.org/10.4000/dhfles.2491

Ce document a été généré automatiquement le 27 mai 2021.

(c) SIHFLES 


\section{André Kaspi (sous la direction de). Histoire de l'Alliance israélite universelle de 1860 à nos jours.}

Paris : Armand Colin, 2010, 575 p. ISBN 978-2-200-35479-4.

\section{Danielle Omer}

1 Cet épais ouvrage (575 pages) est paru, au début de l'année 2010, à l'occasion du $150^{e}$ anniversaire de la création de l'Alliance israélite universelle dont l'importance de l'œuvre éducative n'est plus à rappeler.

2 L'AIU s'est toujours préoccupée de commémorer la date de sa création. Des ouvrages majeurs pour la compréhension de l'action de cette société ont en leur temps paru comme, par exemple, celui de Narcisse Leven, pour le cinquantenaire, Cinquante ans d'histoire: l'Alliance israélite universelle (1860-1910) ou encore celui d'André Chouraqui, pour le centenaire, L'Alliance israélite universelle et la renaissance juive contemporaine. Cent ans d'histoire. En dehors de ces deux dates marquantes, l'AIU a irrégulièrement commandité des ouvrages pour des commémorations intermédiaires, comme celui, encore très proche de nous, de Georges Weill, pour les 140 ans de l'AIU et la commémoration du deuxième millénaire de l'ère moderne, Emancipation et progrès. L'Alliance israélite universelle et les droits de l'homme.

3 Histoire de l'Alliance israélite universelle de 1860 à nos jours a pour particularité d'être un ouvrage collectif et international qui fait appel à des chercheurs français, américains et israéliens afin «de remettre sur le chantier l'histoire des 150 premières années de l'Alliance " (Préface : p. 9) et de "proposer un état de la question» (ibidem) en se fondant sur la documentation disponible et en partie nouvellement accessible. L'ouvrage dirigé par A. Kaspi se propose d'apporter des éclairages nouveaux sur des périodes encore mal connues de l'Alliance, comme par exemple la période du gouvernement de Vichy, ou celle qui a suivi la création d'Israël qui entraîna ce que Kaspi nomme l'acte d'aggiornamento de l'Alliance, correspondant à la prise en compte et à l'acceptation des succès du sionisme politique. 
4 L'ouvrage est divisé en 12 chapitres rédigés par différents chercheurs. Chaque chapitre comporte des extraits de textes d'archives qui illustrent et complètent les contenus des articles ou encore contiennent des notices qui apportent des précisions sur des institutions liées à l'AIU ou des personnalités de l'AIU ou proches d'elle. La plupart de ces compléments sont rédigés ou présentés par un auteur différent de celui de l'article, ce qui permet une lecture à deux entrées, très agréable pour le lecteur qui ne se sent pas ainsi obligé de considérer qu'il a « un pavé » à lire.

5 Les 12 chapitres ont pour intitulé et pour auteur(s):

-ch. 1 : « Aux origines de l'Alliance » par Perrine Simon-Nahum,

-ch. 2 : « Les structures et les hommes » par Georges Weill,

- ch. 3: «Solidarité et défense des droits des juifs (1860-1914)»par Grégoire Kauffmann, Michel M. Laskier et Simon Schwarzfuchs,

- ch. 4 : «La Grande Guerre et ses lendemains » par Dzovinar Kevonian et Philippe Landau,

- ch. 5 : « Face au sionisme (1897-1940)» par Catherine Nicault,

-ch. 6 : «La mission éducative (1860-1939» par Aron Rodrigue,

-ch. 7 : «L'émancipation des femmes » par Frances Malino,

-ch. 8 : «Dans la tourmente de la Seconde Guerre mondiale (1939-1944)» par Catherine Nicault,

- ch. 9 : « Reconstruction et nouvelles orientations » par Anne Grynberg,

-ch. 10 : « L'Alliance en Israël » par Ariel Danan,

- ch. 11: «Décolonisation et indépendances: l'impact en Afrique du Nord» par Michel Abitbol,

- ch. 12 : «Vers une nouvelle Alliance? » par Sophie Enos-Attali.

Des annexes avec des documents importants comme l'appel de 1860, les statuts de 1862, la liste chronologique des membres du bureau du comité central, des données sur le réseau scolaire et des cartes, etc. complètent les 12 contributions.

On le remarque tout de suite, les chapitres traitent chronologiquement l'histoire de l'Alliance, laquelle est, grâce à la pluralité des auteurs et à leur notoriété dans le domaine, assurée d'être exprimée au travers d'une certaine diversité d'opinions permettant d'éviter ainsi le ronron que malheureusement beaucoup d'ouvrages commémoratifs produisent fréquemment.

8 Le premier chapitre cherche à comprendre les ancrages politiques et idéologiques des fondateurs de l'AIU, tout particulièrement ceux d'Adolphe Crémieux qui en fut l'âme et des six autres qui signèrent le manifeste-appel de 1860 puis élaborèrent les statuts de 1862 : Élie-Aristide Astruc, Isidore Cahen, Jules Carvallo, Eugène Manuel, Charles Netter et Narcisse Leven, le plus jeune, qui en deviendra à partir de 1898 le président. Tous sont de fervents héritiers des principes de la Révolution de 1789 qui, entre autres, émancipa pour la première fois en Europe les juifs, et tous sont aussi imprégnés des principes libéraux de progrès et d'émancipation universelle issus de la Révolution de 1848. Ce premier chapitre essaie de comprendre comment ces sept hommes de formation diverse (juristes, polytechnicien, homme de lettres, rabbin, commerçant) ont pu se retrouver et sur quelles bases : la lutte contre l'antisémitisme et les scandales qui l'accompagnent comme par exemple l'affaire Mortara, la défense des juifs opprimés dans le monde et le devoir de solidarité envers les coreligionnaires, l'affirmation des principes de tolérance à l'intérieur des États et l'accès pour tous à la citoyenneté. 
9 Le deuxième chapitre rend compte de l'organisation de l'AIU qui dispose à son sommet d'un comité central, l'organe directeur. Ce comité central recrute ses membres (résidents et non-résidents en France), tout d'abord sur le mode d'une cooptation masquée puis après 1920 par cooptation ouverte afin de ne pas laisser la possibilité à d'autres sensibilités de prendre le contrôle de la direction. À ce propos, le conflit avec les comités allemands est relaté, ce qui aboutit d'une part à la création d'une société allemande concurrente en 1901 (Hilfsverein der Deutschen Juden) et d'autre part, en 1919, à l'éviction des membres allemands du comité central. Les membres du comité central constituent « un cénacle homogène et représentatif de la finance internationale, de la haute bourgeoisie et d'une élite intellectuelle juive, représentée, en France par les universitaires, à l'étranger par les rabbins ». (p. 79) Enfin, l'administration de l'AIU, qualifiée de bureaucratique, est décrite surtout par les notices biographiques des deux figures marquantes que furent les secrétaires Isidore Loeb de 1869 à 1892 et Jacques Bigart de 1892 à 1934, lesquels tenaient toute la correspondance avec tous les directeurs et directrices du réseau scolaire.

10 Le troisième chapitre traite de l'action politique, diplomatique et médiatique que l'AIU déploya entre 1860 et 1914 pour combattre l'antisémitisme hors de France. Sont principalement décrites dans cette contribution les actions entreprises pour que le sort des juifs de Russie et du Maghreb - notamment au Maroc -, très discriminés et objets réguliers de la vindicte populaire, s'améliorât. En parallèle, il est expliqué pourquoi l'AIU resta très discrète lors des crises d'antisémitisme aigu (affaire Dreyfus) que la France traversa. Cette période "peut être considérée comme l'âge d'or de l'action politique et diplomatique de l'organisation ». (p. 155)

11 Le quatrième chapitre concerne la guerre de 1914-1918. Durant ces quatre années, l'AIU, qui défend totalement la cause française tout en tentant de continuer à afficher sa vocation universaliste, cherche à maintenir autant que faire se peut son réseau scolaire. Elle y parvient partiellement en Afrique du Nord, mais les fonds manquent cruellement. Après l'engagement de l'Empire ottoman aux côtés des puissances de l'Axe, le réseau des écoles dans cette région devient pour les autorités ottomanes comme pour les autorités françaises un foyer ennemi. De manière générale, le personnel enseignant ne perçoit plus de salaire régulier, la correspondance avec Paris est pratiquement coupée. Parallèlement, l'antisémitisme virulent renaît dans la presse française, dans les forces armées et par voie de conséquence chez les coloniaux d'Afrique du Nord et sur le territoire métropolitain. Les juifs engagés sont considérés comme des éléments peu sûrs, il est reproché, en outre, aux juifs d'Afrique du Nord de ne pas vouloir faire leur devoir militaire, etc., etc. L'AIU reste très discrète au nom de l'intérêt supérieur patriotique français sur toutes ces manifestations racistes d'expression française. Parallèlement, les organisations juives européennes et américaines de tendance sioniste reprochent à l'AIU son étroit engagement franconational. Après la fin du conflit, l'AIU déploie une intense activité diplomatique pour que les populations juives, dans les nouveaux États créés par les traités, soient assurées d'une égalité de droit et de traitement dans une perspective assimilationniste. De fait, elle doit constater que d'autres organisations juives sionistes ou favorables au sionisme lui contestent sa prétention au leadership et défendent avec succès la reconnaissance du Foyer national juif en Palestine (voir la déclaration Balfour en 1917).

12 Le cinquième chapitre reprend plus spécifiquement la question de l'émergence et de l'avancée du courant sioniste avec T. Herzl. Ce courant correspond à une revendication 
nationaliste moderne : il faut considérer les juifs comme un peuple formant une nation et de ce fait ayant droit à un État. L'AIU, en face, défend une autre conception : la notion de "peuple juif » appartient à un monde ancien et révolu, c'est la confession qui crée les liens de judéité et de solidarité entre les coreligionnaires. La « question juive » se résoudra lorsque les juifs, jouissant des mêmes droits que les autres citoyens, se seront assimilés dans les sociétés où ils sont déjà enracinés. L'Alliance défendra sa conception avec vigueur ; le personnel enseignant suivra très majoritairement, sauf, par exemple, en Bulgarie. L'Alliance restera plutôt discrète sur ses vives querelles avec le courant sioniste afin de ne pas, pense-t-elle, lui donner de l'audience. Cependant les organisations juives allemandes et américaines, sionistes ou sympathisantes, gagnent du terrain sur tous les plans : financier, diplomatique et politique. La France, ellemême, soutient, par calcul politique, la déclaration Balfour. L'AIU perd cette bataille et est obligée de changer d'orientation après la Deuxième Guerre mondiale, la Shoah et la création d'Israël créant les conditions de ce revirement. objectifs assignés à "la mission éducative » du réseau scolaire entre 1860 et 1939 et comment celui-ci était conçu et organisé. Il est expliqué comment la création de toute école repose sur une demande faite par la communauté concernée et comment l'AIU apporte sa contribution: envoi de directeurs formés à l'École normale israélite orientale (ENIO) et rémunérés par l'Alliance, octroi d'une subvention. La communauté, par l'intermédiaire d'un comité scolaire créé ad hoc, gère l'école en s'occupant de prélever l'écolage, de récolter des fonds pour le fonctionnement, de rémunérer les enseignants vacataires, de mettre le plus souvent un local à disposition. Ces établissements ont pour objectif d'occidentaliser la jeunesse juive, de la "régénérer » selon l'expression utilisée fréquemment dans les textes, en proposant une éducation plus qu'une instruction. Il faut, pour l'AIU, en plus d'une instruction de base (lire, écrire et calculer) détruire ce qu'elle considère comme une "arriération » qui empêche les juifs de ces communautés de prendre leur destin en main. C'est une entreprise de «moralisation» dans laquelle s'est engagée l'Alliance. Les instituteurs formés à Paris restaient en contact épistolaire très étroit avec l'administration centrale et rendaient compte de la bonne marche de l'école et de tous les faits marquants dans la communauté. Tous étaient de fervents défenseurs du programme éducatif de l'Alliance.

14 Parmi les spécificités de ce réseau scolaire, on compte la création d'un grand nombre d'établissements pour la formation professionnelle dans le domaine de l'artisanat et de l'agriculture. Beaucoup d'efforts furent consentis en ce sens, bien que les résultats n'eussent pas toujours été à la hauteur.

Une autre spécificité réside dans l'importance accordée à l'éducation juive et religieuse. Ceci répond aux exigences des communautés qui avaient la charge du recrutement et de la rémunération des enseignants-rabbins concernés et pour qui l'instruction religieuse était très souvent une impérieuse nécessité, sinon la seule. L'Alliance de son côté souhaitait une éducation juive "de qualité » et imposa à partir de 1892 un cours d'histoire juive post-biblique qui sortait ainsi les enfants de l'étroit cadre religieux biblique. Cet enseignement fut presque toujours un sujet de très graves discordes entre les enseignants de l'Alliance et les juifs traditionalistes et, plus tard, les sionistes.

16 Enfin, la langue française resta pratiquement partout la seule langue de scolarisation, l'enseignement des langues locales véhiculaires étant prévu dans le programme mais restant négligé et même parfois absent. 
17 Le septième chapitre est consacré à la scolarisation des filles. "L'Alliance avait une multitude d'objectifs à l'esprit quand elle établit ses écoles de filles. Il s'agissait d'une part de sauver les jeunes élèves de la prostitution, de l'esclavage domestique, ou d'un mariage précoce.» (p. 266) Ces écoles ont eu pour mission de former des jeunes filles qui puissent ensuite exercer leur rôle de mère de famille de telle sorte qu'il soit en résonance avec le niveau d'éducation moderne reçu par leur futur époux. En cela, l'Alliance accompagnait les conceptions françaises de la métropole. Ces jeunes femmes furent « des vecteurs d'occidentalisation et les catalyseurs d'un changement culturel. Ainsi leur influence s'étendit bien au-delà de leur propre foyer. » (p. 293)

Le huitième chapitre aborde la période très méconnue (perte des archives) du devenir de l'AIU durant la Seconde Guerre mondiale. La défaite de 1940, l'exode qui s'ensuit et l'occupation de Paris par les forces nazies désorganisent profondément l'institution de l'Alliance, déjà affaiblie par la perte d'un grand nombre d'écoles dans la Turquie kémaliste et l'avancée du sionisme. Bon gré mal gré, l'AIU se replie à Vichy et est incorporée à l'Union générale des israélites de France (1942), organisation créée de toutes pièces par le gouvernement de Vichy. "À l'instar de la plupart des notables israélites, les hommes de l'Alliance ont été des 'maréchalistes', longtemps incapables de croire la France de Pétain directement responsable de la politique antisémite.» (p. 318-319) Malgré le soutien financier et plutôt en sous-main du Service des Euvres françaises à l'étranger, rattaché au Quai d'Orsay, le réseau scolaire est désorganisé et livré à lui-même. Il faudra attendre que René Cassin, rallié à la France combattante depuis Londres, soit investi, en 1943 par De Gaulle, de la mission de « redonner vie à l'Alliance en déshérence [...] » (p. 325) René Cassin deviendra vite président de l'AIU et le restera jusqu'à sa mort en 1976.

Le neuvième chapitre retrace les efforts de réorientation du positionnement idéologique de l'AIU et de reconstruction du réseau scolaire, immédiatement après la guerre. Sous la direction de R. Cassin, « se met en place une structure d'encadrement élargie au sein de laquelle trois groupes se dessinent : 'les anciens notables, les recrues de la guerre, celles de la Libération'. » (p. 332) Concrètement, il y a beaucoup à faire : les finances sont exsangues, des biens immobiliers spoliés doivent être récupérés, les ouvrages de la bibliothèque pillée doivent être rapatriés. Enfin le positionnement idéologique de l'Alliance doit être repensé. Pendant des mois, d'âpres discussions ont lieu pour savoir si l'AIU ralliera le Conseil représentatif des juifs de France (CRIF), fondé en 1944 dans la clandestinité. Si oui, l'AIU craint ainsi d'apparaître comme un groupement de juifs en France et non plus d'être un groupe de Français de confession juive comme cela avait toujours été le cas. De plus, l'Alliance craint de perdre ainsi sa visibilité. Finalement l'adhésion est votée à la majorité des voix. Parallèlement, un programme d'action (déclaration du 11 novembre 1945, donnée en annexe) est élaboré, dans lequel la mission éducative de l'Alliance est réaffirmée, et la libre entrée des juifs immigrants en Palestine est demandée.

20 Afin de pouvoir reprendre ses activités éducatives et son réseau scolaire, l'AIU est contrainte de nouer de nouvelles alliances, notamment avec l'American Jewish Joint Distribution Committee pour renflouer ses finances et reconstituer ses réseaux.

Le dixième chapitre montre comment l'AIU, qui, après 1946, soutient très vite et sans faille le sionisme politique puis l'État d'Israël, cherche alors à s'implanter en Israël audelà de ses établissements traditionnels que sont l'école des sourds-muets de Jérusalem et la ferme-école de Mikveh Israël. L'Alliance, contrainte dès 1951 de rendre ses écoles 
élémentaires, cherche à créer des établissements qui scolarisent les juifs pauvres d'Afrique du Nord. Elle crée aussi des établissements secon-daires gratuits à Jérusalem, Tel-Aviv et Haïfa où l'enseignement du français est privilégié. Malgré la qualité de l'enseignement, les succès sont mitigés. Au total, de nombreux projets ont été proposés, quelques-uns ont été réalisés et seuls certains ont été couronnés de succès, l'enseignement du français et en français attirant un public peu nombreux.

Le onzième chapitre décrit comment, à la veille des indépendances au Maghreb, les écoles de l'Alliance sont arabisées et intégrées sans préalable aucun en Tunisie, comment les juifs algériens, de nationalité française depuis le décret Crémieux, sont en passe d'être abandonnés par la France et revendiqués comme citoyens algériens par les mouvements de lutte pour l'indépendance. Le Maroc fait figure d'exception jusqu'en 1959 environ. L'école normale hébraïque de Casablanca est créée en 1951, elle forme des enseignants pour l'hébreu. Des écoles nouvelles sont créées, des sections qui prolongent les études sont rajoutées dans les écoles déjà existantes. Le réseau scolaire de l'Alliance y est florissant jusqu'en 1958, moment où le parti de l'Istiqlal arrivant au pouvoir impose l'arabisation et l'ouverture des écoles à tous quelle que soit la confession. Au bout du compte, les juifs du Maghreb, souffrant de la dégradation de leurs conditions de vie, partent pour Israël, la France et l'Amérique du Nord.

Le douzième chapitre s'efforce de faire le panorama des activités actuelles de l'AIU. Elle poursuit toujours sa mission éducative et se trouve à la tête d'un nouveau réseau scolaire redéployé en France, en Europe, en Israël et au Canada principalement, combinant la direction directe d'écoles et le partenariat. La bibliothèque, les archives, le Collège des études juives, des activités éditoriales constituent les autres points forts de son activité. L'Alliance est devenue le centre français de la connaissance du monde juif. Mais l'AIU souffre toujours d'un déficit financier chronique qui l'oblige, sans cesse, à se mettre en quête de financements, de dons et de subventions.

\section{AUTEUR}

\section{DANIELLE OMER}

Université du Mans 